\title{
Explanation of the Optical Spectra and Spin-Hamiltonian Parameters for Nickel(II) in Cadmium Bromide Crystal
}

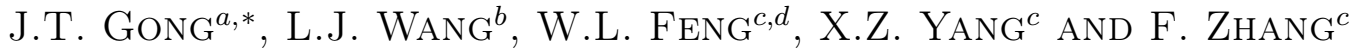 \\ ${ }^{a}$ School of Physics and Electron, Mianyang Normal University, Mianyang, 621000, China \\ ${ }^{b}$ Department of Physics and Electronic Engineering, Neijiang Normal College, Neijiang, 641112, China \\ ${ }^{c}$ Department of Applied Physics, Chongqing University of Technology, Chongqing, 400054, P.R. China \\ ${ }^{d}$ International Centre for Materials Physics, Chinese Academy of Sciences, Shenyang, 110016, P.R. China
}

(Received June 28, 2010)

\begin{abstract}
Based on crystal- and ligand-field theory, double-spin-orbital coupling approach was used to analyze the crystal-field energy levels and spin-Hamiltonian parameters of $\mathrm{Ni}^{2+}$ ion at trigonal site in $\mathrm{CdBr}_{2}$. The local lattice distortion $\left(\Delta R\right.$ and $\left.\tau_{\mathrm{Ni}^{2+}}\right)$ is estimated from the crystal field parameters; the crystal field energy Hamiltonian was diagonalized in the full basis consisting of 45 wave functions of the $\mathrm{Ni}^{2+}$ ion. Results of calculations are in good agreement with experimental data. The reasonableness of the theoretical results is discussed.
\end{abstract}

PACS: 71.70.Ch, 75.10.Dg, 61.72.Bb, 76.30.Fc

\section{Introduction}

$\mathrm{CdBr}_{2}$ is an important metal dihalide compound in which the $\mathrm{Cd}^{2+}$ plane is sandwiched between two $\mathrm{Br}^{-}$ anions planes (see Fig. 1) [1, 2]. Its crystal structure is rhombohedra and it belongs to the space group $D_{3 d}^{5}[1,2]$. Because there are good transparent and dielectric characteristics of $\mathrm{CdBr}_{2}$ crystal, various theoretical and experimental methods were used to study its physical and chemical properties and microstructure [3-5]. The optical and electron paramagnetic resonance (EPR) spectra [i.e., the spin-Hamiltonian (SH) parameters including the anisotropic $g$ factors and zero-field splitting $D$ ] of $\mathrm{CdBr}_{2}: \mathrm{Ni}^{2+}$ was measured for the substitutional $\mathrm{Ni}^{2+}$ center $[4,5]$. Since the SH parameters of paramagnetic ions depend sensitively on the structural parameters $R$ and $\theta$, it will be useful to determine these parameters with a transition-metal ion as the probe. The EPR study of $\mathrm{Ni}^{2+}$ ions in $\mathrm{CdBr}_{2}$ has been done by Fang et al. [6]. They calculated the EPR parameters with the high-order perturbation formulae. As much as we know, however, there is no unified theoretical analysis for both optical and EPR spectra of these experimental data of $\mathrm{CdBr}_{2}: \mathrm{Ni}^{2+}$ crystal. The lattice structure data of these impurity centers have not been acquired yet by the unified calculation method. In fact, since the spin-orbit coupling coefficient of the ligand $\mathrm{Br}^{-}$(e.g. $\left.\zeta_{p}^{0}=2460 \mathrm{~cm}^{-1}[7]\right)$ is bigger than that of the central

* corresponding author; e-mail: gongjt08@163.com
$\mathrm{Ni}^{2+}$ (e.g. $\zeta_{d}^{0}=640 \mathrm{~cm}^{-1}[8]$ ), the ligand influence should be included in the analysis of the optical and EPR spectra data. In this work, the full energy matrix based on the basis consisting of 45 wave functions of the $\mathrm{Ni}^{2+}$ ion are established and then applied to $\mathrm{Ni}^{2+}$ center in $\mathrm{CdBr}_{2}$ crystal. In the calculations, the contributions from the ligands $p$ orbitals and the local angle distortions due to the mismatch of ionic radius between the host and impurity are taken into account with the double-spin-orbit coupling approach.

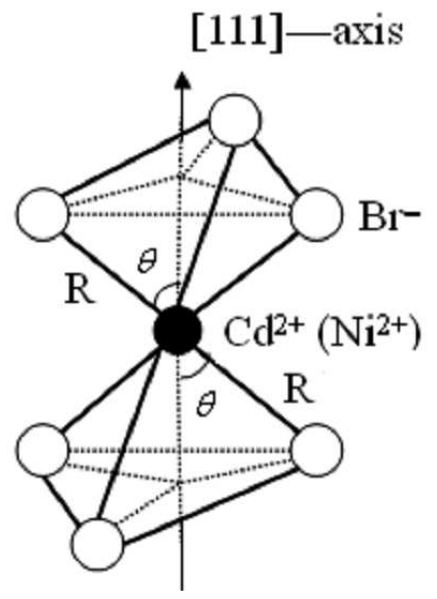

Fig. 1. The local structure around $\mathrm{Ni}^{2+}$ centers in $\mathrm{CdBr}_{2}: \mathrm{Ni}^{2+}$ crystals, including the angle $\theta$ used in the calculation. 


\section{Calculations}

\subsection{Double-spin-orbital coupling approach model}

According to the molecular orbital (MO) theory, the single electron wave function can be reasonably well described by the following form:

$$
|\gamma\rangle=N_{\gamma}\left(\left|d_{\gamma}\right\rangle-\lambda_{\gamma}\left|p_{\gamma}\right\rangle\right)
$$

where $\gamma=t_{2 \mathrm{~g}}$ or $e_{\mathrm{g}}$ is the irreducible representation of the $O_{h}$ group. $\left|d_{\gamma}\right\rangle$ is a metal orbital and $\left|p_{\gamma}\right\rangle$ is a symmetry-adapted linear combination of valence orbital of the involved ligand. $N_{\gamma}$ and $\lambda_{\gamma}$ are, respectively, the normalization coefficients and the mixing coefficients of the atomic orbits.

Using the double-spin-orbit coupling approach, the spin-orbit coupling operator for two-electron Hamiltonian $\left(H_{\mathrm{SO}}\right)$ is taken as

$$
H_{\mathrm{SO}}=\sum_{i} \zeta_{d}\left(r_{d}\right) l_{i} s_{i}+\sum_{i} \zeta_{p}\left(r_{p}\right) l_{j} s_{j}
$$

Here the first term corresponds to spin-orbit coupling effect of the central ion while the second term refers to that of the ligand.

The MO coefficients $N_{\gamma}$ and $\lambda_{\gamma}$ can be estimated by a semi-empirical method [9]. According to this method, the approximation relation and the normalization correlation can be given as

$$
\begin{aligned}
& B / B_{0} \approx N_{\gamma}^{2}\left[1-2 \lambda_{\gamma} S_{d p}(\gamma)+\lambda_{\gamma}^{2} S_{d p}^{2}(\gamma)\right], \\
& N_{\gamma}\left[1-2 \lambda_{\gamma} S_{d p}(\gamma)+\lambda_{\gamma}^{2}\right] \approx 1,
\end{aligned}
$$

where $B / B_{0}$ is the rate between the electrostatic Coulomb energy of the transition-metal ions in the crystal and of the free ions. $S_{d p}(\gamma)$ is the group overlap integral of $d_{\gamma}$ and $p_{\gamma}$ orbitals.

\subsection{The full energy matrix and EPR (SH parameters) formulae}

For a $3 d^{8}$ ion in a trigonal crystal field, the full energy Hamiltonian can be written as

$$
\begin{aligned}
H & =H_{\mathrm{ee}}(B, C)+H_{\mathrm{CF}}(D q, D \tau, D \sigma) \\
& +H_{\mathrm{SO}}\left(\zeta_{+}, \zeta_{-}\right),
\end{aligned}
$$

where $H_{\mathrm{ee}}(B, C), H_{\mathrm{CF}}(D q, D \tau, D \sigma)$ and $H_{\mathrm{SO}}\left(\zeta_{+}, \zeta_{-}\right)$, are the electrostatic interaction, $B$ and $C\left(\approx B C_{0} / B_{0}\right.$, $B_{0} \approx 1084 \mathrm{~cm}^{-1}$ and $C_{0} \approx 4831 \mathrm{~cm}^{-1}$ [8]) are the Racah electrostatic parameters characterized with the electrostatic Coulomb interaction as mentioned above, the crystal field and the spin-orbit Hamiltonian of the central-metal ion $(3 d)$ and the ligand ion $(4 p)$ interactions, respectively.

Based on the double-spin-orbit coupling approach, the two spin-orbit parameters $\zeta_{+}, \zeta_{-}$and two orbital reduction factors $k_{+}, k_{-}$can be written as $[10,11]$ :

$$
\begin{aligned}
& \zeta_{+}=N_{t}\left(\zeta_{d}^{0}+\lambda_{t}^{2} \zeta_{p}^{0} / 2\right), \\
& \zeta_{-}=\left(N_{t} N_{e}\right)^{1 / 2}\left(\zeta_{d}^{0}-\lambda_{t} \lambda_{e} \zeta_{p}^{0} / 2\right),
\end{aligned}
$$

$$
\begin{aligned}
& k_{+}=N_{t}\left[1-2 \lambda_{t} S_{d p}\left(t_{2 \mathrm{~g}}\right)+\lambda_{t}^{2} / 2\right], \\
& k_{-}=\left(N_{t} N_{e}\right)^{1 / 2} \\
& \quad \times\left[1-\lambda_{t} S_{d p}\left(t_{2 \mathrm{~g}}\right)-\lambda_{e} S_{d p}\left(e_{\mathrm{g}}\right)-\lambda_{t} \lambda_{e} / 2\right] .
\end{aligned}
$$

Considering the equivalence between the $\mathrm{SH}$ parameters and the Zeeman terms, we have

$$
\begin{aligned}
& D=E\left(\left|t_{2}^{6} e^{2}{ }^{3} A_{2} \pm 1 e_{2}\right\rangle^{\prime}\right)-E\left(\left|t_{2}^{6} e^{2}{ }^{3} A_{2} 0 e_{2}\right\rangle^{\prime}\right), \\
& g_{\|}=\left\langle\left. t_{2}^{6} e^{2}{ }^{3} A_{2} 1 e_{2}\right|^{\prime} k_{ \pm} L_{z}+g_{s} S_{z} \mid t_{2}^{6} e^{2}{ }^{3} A_{2} 1 e_{2}\right\rangle^{\prime}, \\
& g_{\perp}=\sqrt{2}\left\langle\left. t_{2}^{6} e^{2}{ }^{3} A_{2} 0 e_{2}\right|^{\prime} k_{ \pm} L_{x}+g_{s} S_{x} \mid t_{2}^{6} e^{2}{ }^{3} A_{2} 1 e_{2}\right\rangle^{\prime},
\end{aligned}
$$

where $\left|{ }^{3} A_{2}\left(t_{2}^{6} e^{2}\right), M_{s}\right\rangle^{\prime}$ and $\left.E\left(\left.\right|^{3} A_{2}\left(t_{2}^{6} e^{2}\right), M_{s}\right\rangle^{\prime}\right)$ are, respectively, the eigenfunction and eigenvalue of the ground state ${ }^{3} A_{2}$ with spin $M_{s}$ obtained by diagonalizing the above complete energy matrix. $L_{j}(j=x, y, z)$ and $S_{j}$ represent the operators of orbit and spin angular momenta. $g_{s}(\approx 2.0023)$ is only the spin value.

The trigonal field parameters $D \tau$ and $D \sigma$ in the energy matrix are calculated from the Newman superposition model [12]. From the model, for the trigonal $d^{n}$ ions in octahedral, we have

$$
\begin{aligned}
D \tau & =-\frac{2}{7} \bar{A}_{4}(R) \\
\times & \left(35 \cos ^{4} \theta-30 \cos ^{2} \theta+3+7 \sqrt{2} \sin ^{3} \theta \cos \theta\right), \\
D \sigma & =-\frac{6}{7} \bar{A}_{2}(R)\left(3 \cos ^{2} \theta-1\right),
\end{aligned}
$$

in which the intrinsic parameters $\bar{A}_{2}(R)$ and $\bar{A}_{4}(R)$ are related to the reference distance $R$. $R$ value can be estimated from the empirical formula $R \approx R_{h}+\left(r_{i}-r_{h}\right) / 2$ $[13,14]$. From literature $[1,15]$, we have the bond length of the host crystal $\left(R_{h} \approx 2.71 \AA\right)$, the radius of the impurity $\mathrm{Ni}^{2+}\left(r_{i}\left(\mathrm{Ni}^{2+}\right) \approx 0.69 \AA\right)$, the radius of the host ion $\mathrm{Cd}^{2+}\left(r_{h}\left(\mathrm{Cd}^{2+}\right) \approx 0.97 \AA\right)$, thus, $R \approx 2.57 \AA$ can be obtained. For a $d^{n}$ ion in octahedral complex, $\bar{A}_{4}\left(R_{0}\right) \approx(3 / 4) D q[16-19]$, where $D q$ is the cubic field parameter which is often obtained from the optical spectra of the studied system. $D q \approx 640 \div 720 \mathrm{~cm}^{-1}$ [4] is found for $\left(\mathrm{NiBr}_{6}\right)^{4-}$ octahedra in crystals, we take $D q \approx 660 \mathrm{~cm}^{-1}$ here. By studying the optical and EPR spectra for $3 d^{n}$ ions in many crystals, the ratio $\bar{A}_{2}(R) / \bar{A}_{4}(R)$ is found to be in the range of $8 \div 12[16-21]$, we take $\bar{A}_{2}(R) / \bar{A}_{4}(R)=9$. The group overlap integrals $S_{d p}\left(e_{\mathrm{g}}\right) \approx 0.0269332$ and $S_{d p}\left(t_{2 \mathrm{~g}}\right) \approx 0.0079138$ are obtained from the Slater-type SCF functions [22, 23] and the metal-ligand distance $R$.

Thus, in the full energy matrix of $3 d^{8}$ electronic configurations, there are only two unknown or adjustable parameters $\theta$ and $B$. They can be obtained by matching the calculated optical spectra and SH parameters $\left(g_{\|}, g_{\perp}\right.$, and $D$ ) to the experimental values. From the calculations using the full energy matrix with the double-spin-orbit coupling approach, we obtain for $\left(\mathrm{NiBr}_{6}\right)^{4-}$ cluster in $\mathrm{CdBr}_{2}: \mathrm{Ni}^{2+}$ crystal 


$$
\theta \approx 54.814^{\circ}, \quad B \approx 832 \mathrm{~cm}^{-1} .
$$

The coefficient $N_{\gamma}$ and the parameters in Eq. (3) calculated from the above $\lambda_{\gamma}$ are collected in Table I. The comparison between the calculated and experimental optical spectra and SH parameters are shown in Table II and Table III.
TABLE II

The $\mathrm{SH}$ parameters of $\mathrm{CdBr}_{2}: \mathrm{Ni}^{2+}$ crystal.

\begin{tabular}{c|c|c|c}
\hline \hline & $g_{\|}$ & $g_{\perp}$ & $D\left[\mathrm{~cm}^{-1}\right]$ \\
\hline Calc. $^{a}$ & 2.2797 & 2.2773 & -0.3246 \\
Calc. $^{b}$ & 2.1900 & 2.1881 & -0.2200 \\
Expt. [5] & $2.19(2)$ & $2.18(2)$ & $0.220(5)$ \\
\hline
\end{tabular}

${ }^{a}$ Taking no account of the effect of the ligand.

${ }^{b}$ Taking account of the effect of the ligand.

The group overlap integral, the molecular orbital coefficients and orbital reduction factors for $\mathrm{CdBr}_{2}: \mathrm{Ni}^{2+}$ crystal.

\begin{tabular}{c|c|c|c|c|c|c|c}
\hline \hline$S_{d p}\left(e_{\mathrm{g}}\right)$ & $S_{d p}\left(t_{2 \mathrm{~g}}\right)$ & $N_{t}$ & $N_{e}$ & $\lambda_{t_{2 \mathrm{~g}}}$ & $\lambda_{e_{\mathrm{g}}}$ & $k_{+}$ & $k_{-}$ \\
\hline 0.0269332 & 0.0079138 & 0.8787 & 0.8853 & 0.3795 & 0.3878 & 0.9367 & 0.8053
\end{tabular}

The energy levels (or optical spectra) of $\mathrm{CdBr}_{2}: \mathrm{Ni}^{2+}$ crystal.

TABLE III

\begin{tabular}{|c|c|c|c|c|c|c|c|}
\hline \multicolumn{2}{|c|}{ Levels } & \multicolumn{2}{|c|}{ Energy $\left[\mathrm{cm}^{-1}\right]$} & \multicolumn{2}{|c|}{ Levels } & \multicolumn{2}{|c|}{ Energy $\left[\mathrm{cm}^{-1}\right]$} \\
\hline Cubic & $D_{3}$ & Cal. & Expt. [4] & Cubic & $D_{3}$ & Cal. & Expt. [4] \\
\hline$t^{6}{ }_{2} e^{23} A_{2}$ & $E$ & 0 & 0 & $t^{5}{ }_{2} e^{3}{ }^{1} T_{2}$ & $A$ & 19342 & 19200 \\
\hline & $A$ & -0.2200 & & & $E$ & 19374 & \\
\hline$t^{5}{ }_{2} e^{33} T_{2}$ & $E$ & 6312 & & $t^{5}{ }_{2} e^{3}{ }^{3} T_{1}$ & $E$ & 21081 & \\
\hline & $A$ & 6399 & & & $E$ & 21334 & \\
\hline & $E$ & 6408 & $6645-7200$ & & $A$ & 21369 & $20580-22200$ \\
\hline & E & 6812 & & & $A$ & 21391 & \\
\hline & $A$ & 6819 & & $t^{6}{ }_{2} e^{2}{ }^{1} A_{1}$ & $A$ & 21423 & \\
\hline & $A$ & 7014 & & $t^{5}{ }_{2} e^{33} T_{1}$ & $E$ & 21465 & \\
\hline$t^{4}{ }_{2} e^{43} T_{1}$ & $A$ & 10478 & & & $A$ & 21476 & \\
\hline & $A$ & 10879 & & $t^{5}{ }_{2} e^{3{ }^{1}} T_{1}$ & $A$ & 24072 & \\
\hline & E & 10896 & $10616-11416$ & & $E$ & 24079 & \\
\hline & E & 11445 & & $t^{4}{ }_{2} e^{4}{ }^{1} E$ & $E$ & 28745 & \\
\hline & E & 11530 & & $t^{4}{ }_{2} e^{4}{ }^{1} T_{2}$ & $E$ & 29144 & \\
\hline & $A$ & 11543 & & & $A$ & 29188 & \\
\hline$t^{6}{ }_{2} e^{2}{ }^{1} E$ & $E$ & 13716 & $11820-13210$ & $t^{4}{ }_{2} e^{4}{ }^{1} A_{1}$ & $A$ & 53630 & \\
\hline
\end{tabular}

\section{Discussions and results}

(a) If we assume that the $S_{d p}(\gamma)=0, \lambda_{\gamma}=0$ and $\zeta_{p}=0$, which the case is for the single-spin-orbit coupling, from Table II, the calculation ${ }^{a}$ shows that the theoretical results are in poor agreement with the experimental values if without regard to the effect of the ligand. In fact, the mixing parameter $\lambda_{\gamma}$ (about 0.38) in Table I also shows that the contribution from the ligand cannot be omitted.

(b) From Table II and III, the sign of zero-field splitting $D$ is suggested as negative. According to the approximate relation $D=-\frac{\zeta_{d}}{4}\left(g_{\|}-g_{\perp}\right)$ for $3 d^{8}$ electron configurations in trigonal symmetry [24], the $\zeta_{d}>0$ and $g$-shift $\Delta g=$ $g_{\|}-g_{\perp}>0$, thus, the negative sign of $D$ can be regarded as reasonable.

(c) Because the ionic radius and electronegative property of $\mathrm{Ni}^{2+}$ are different from those of the $\mathrm{Cd}^{2+}$, therefore the $\mathrm{Ni}^{2+}$ in the bromine octahedral has a small change of the lattice structure. The shift of bond length $\Delta R\left(\approx R-R_{h}\right) \approx-0.14 \AA$, and bond angle distortion $\tau_{\mathrm{Ni}^{2+}}\left(\approx \theta-\theta_{h}=54.814-57.30^{\circ}[1]\right) \approx-2.486^{\circ}$ can be obtained from the investigation. This case shows that the $\mathrm{CdBr}_{2}: \mathrm{Ni}^{2+}$ crystal possesses a compressed distortion comparing with the host $\mathrm{CdBr}_{2}$. 


\section{References}

[1] H. Shiwaku, Y. Okamoto, T. Yaita, S. Suzuki, K. Minato, H. Tanida, Z. Naturforsch. 60a, 81 (2005).

[2] S.D. Sharma, G.L. Sharma, V.K. Agrawal, Acta Crystallogr. B 36, 26 (1980).

[3] M. Fujita, H. Nakagawa, H. Matsumoto, T. Miyanaga, M. Watanabe, K. Fukui, E. Ishiguro, Y. Fujii, Y. Sakisaku, J. Phys. Soc. Jpn. 59 338 (1990).

[4] J. Ackerman, C. Fouassier, E.M. Holt, S.L. Holt, Inorg. Chem. 11, 3118 (1972).

[5] K. Koichi, T. Yasaburo, J. Phys. Soc. Jpn. 28, 1368 (1970).

[6] W. Fang, X.X. Wu, W.C. Zheng, J. Magn. Magn. Mater. 320, 2784 (2008).

[7] G.L. Mcpherson, R.C. Koch, G.D. Stacky, J. Chem. Phys. 60, 1424 (1974).

[8] J.S. Griffith, The Theory of Transition-Metal Ions, Cambridge University Press, London 1964.

[9] M.L. Du, M.G. Zhao, Phys. Status Solidi B 153, 249 (1989).

[10] M.L. Du, C. Rudowicz, Phys. Rev. B 46, 8974 (1992).

[11] W.C. Zheng, Y.J. Fan, X.X. Wu, Z. Naturforsch. A 60, 433 (2005).

[12] D.J. Newman, B. Ng, Rep. Prog. Phys. 52, 699 (1989).
[13] W.C. Zheng, Physica B 215, 255 (1995).

[14] Z.M. Li, W.L. Shuen, J. Phys. Chem. Solids 57, 1073 (1996).

[15] R.C. Weast, CRC Handbook of Chemistry and Physics, CRC Press, Boca Raton 1989, p. F-187.

[16] C. Rudowicz, Y.Y. Zhou, J. Magn. Magn. Mater. 111, 153 (1992).

[17] W.C. Zheng, X.X. Wu, Q. Zhou, Y. Mei, Spectrochim. Acta A 66, 126 (2007)

[18] A. Edgar, J. Phys. C 9, 4303 (1976).

[19] T.H. Yeom, S.H. Choh, M.L. Du, M.S. Tang, Phys. Rev. B 53, 3415 (1996).

[20] W.L. Feng, W.Q. Yang, W.C. Zheng, H.G. Liu, Physica B 405, 2018 (2010)

[21] W.L. Feng, X.X. Wu, W.C. Zheng, Phys. Status Solidi B 244, 3308 (2007).

[22] E. Clementi, D.L. Raimondi, J. Chem. Phys. 38, 2686 (1963)

[23] E. Clementi, D.L. Raimondi, W.P. Reinhardt, J. Chem. Phys. 47, 1300 (1967).

[24] A.K. Petrosyan, A.A. Mirzakhanyan, Phys. Status Solidi $B$ 133, 15 (1986). 